\title{
Conteúdo volumétrico da água no solo via modelos de competição interespecífica
}

\author{
Wilian Jeronimo dos Santos $^{1}$, Rosane Ferreira de Oliveira ${ }^{1}$, Marcos Bacis \\ Ceddia $^{2} \&$ Juliana Lima de Almeida ${ }^{1}$
}

(1) Universidade Federal Rural do Rio de Janeiro, Departamento de Matemática, Rodovia BR 465, Km 07 S/N, Zona Rural 23897-000, Seropédica, Rio de Janeiro, Brasil. E-mail: wilianj@coc.ufrj.br, rosanef@ufrrj.br

(2) Universidade Federal Rural do Rio de Janeiro, Departamento de Solos, Rodovia BR 465, Km $07 \mathrm{~S} / \mathrm{N}$, Zona Rural 23890-000, Seropédica, Rio de Janeiro, Brasil. E-mail: ceddia@ufrrj.br

Santos W.J., Oliveira R.F., Ceddia M.B. \& Almeida J.L. (2018) Conteúdo volumétrico da água no solo via modelos de competição interespecífica. Pesquisa e Ensino em Ciências Exatas e da Natureza, 2(edição especial): 30-39. http://dx.doi.org/10.29215/pecen.v2i2.1038

Editor acadêmico: Angel Ramon S. Delgado. Recebido: 24 Abril 2018. Aceito: 16 Maio 2018. Publicado: 26 Novembro 2018.

Resumo: $\mathrm{O}$ objetivo do presente trabalho é obter uma ferramenta que possa contribuir para a predição da umidade no solo, considerando um solo heterogêneo e bem drenado, para um determinado período de tempo. No balanço hídrico, como critério de redistribuição da água no solo é feita uma analogia com o modelo de competição em dinâmica de populações. Esta analogia pode ser explicada observando o movimento da água no solo a partir de uma "competição" pela água entre os diferentes compartimentos do solo. Esta competição pela água ocorre na prática pela diferença de potencial hidráulico em cada profundidade de um perfil de solo. Dessa forma, a modelagem proposta é baseada nos modelos de competição interespecífica. Além disso, a metodologia apresentada se caracteriza por possuir uma solução numérica mais estável e mais eficiente do que a tradicional e altamente não-linear equação de Richards. Resultados numéricos foram comparados a partir de resultados obtidos pelo programa Hydrus-1D.

Palavras chave: Modelagem matemática, equação de Richards, simulação numérica, recursos hídricos.

\section{Volumetric soil water content by interspecific competition models}

Abstract: The present work aimed to create a tool that can contribute to the prediction of moisture content in heterogeneous and well-drained plots of soil over a given period of time. To characterise the water balance, which is a criterion for the redistribution of water in the soil, an analogy is made to the competition model in population dynamics. This analogy can be explained by "competition" for water among the different soil compartments. The different layers "compete" with each other for water due to difference in hydraulic potential. In this sense, the model proposed is based on models of interspecific competition. Furthermore, the numerical methodology proposed is characterized to be solved more easily than the traditional and highly non-linear Richards' equation. Numerical results were compared against simulation results from the Hydrus-1D program.

Key words: Mathematical modelling, Richards' equation, numerical simulation, water resources.

\section{Introdução}

A predição do movimento da água em solos insaturados é fundamental em diversos ramos da ciência e da engenharia, tais como hidrologia, engenharia ambiental e ciências do solo. $\mathrm{Na}$ agricultura, por exemplo, a água do solo é essencial para o crescimento das plantas e é o 
veículo para o transporte de solutos, incluindo nutrientes e contaminantes no solo (Dardanelli 2004).

Segundo Reichardt \& Timm (2004), o conteúdo de água armazenado no solo em um dado instante é função do balanço hídrico, o qual é o produto do balanço da entrada de água (chuva e/ou irrigação) e saída (escorrimento superficial, drenagem interna por ação da gravidade e evapotranspiração). Tal conteúdo é importante para a eficiência com que são usados os recursos para o cultivo de culturas agrícolas, sendo um parâmetro essencial dos modelos matemáticos que representam uma viabilidade agrícola sustentável, através de um equacionamento das condições de produção e receita líquida esperada com recursos limitados (Vale et al. 2016).

A umidade do solo pode ser estimada experimentalmente, no entanto, para estudos com necessidade de monitoramento em grandes áreas e em alta frequência, os métodos de medição apresentam limitações associadas a aspectos inerentes a seu princípio teórico, precisão, custos e tempo envolvido. Desta forma, o desenvolvimento de modelos de simulação da umidade do solo é fundamental, pois permitem fazer estimativas em grandes áreas e com maior rapidez. Por outro lado, a qualidade dos modelos de simulação é altamente dependente de uma correta validação e determinação de atributos do solo que interferem na dinâmica da água no solo (Bornhoft 1994).

A equação de Richards (Richards 1931) descreve o movimento da água num meio poroso saturado/insaturado e, em geral, não possui solução analítica. $\mathrm{O}$ método das diferenças finitas (Celia et al. 1990), o método dos elementos finitos (Forsyth et al. 1995) e, mais recentemente, o método dos volumes finitos (Eymard et al. 1999; Takeuchi et al. 2010; Zambra et al. 2012) têm sido usados para resolver numericamente a equação de Richards. No entanto, uma solução numérica robusta, capaz de propor acurácia e eficiência para problemas multidimensionais, continua sendo um desafio, devido o comportamento altamente não-linear da equação de Richards (Lai \& Ogden 2015). Em geral, os aspectos analisados dos métodos numéricos incluem: conservação de massa, estabilidade, acurácia, eficiência, continuidade a partir do regime insaturado para o saturado (saturação variável) e a não linearidade da condutividade hidráulica (Caviedes-Voullieme et al. 2013).

Diversos trabalhos na literatura fazem referência ao programa Hydrus ${ }^{\circledR}$ (Simunek et al. 1999). Tal programa simula o movimento da água num meio poroso saturado/insaturado, podendo ser aplicado num perfil (2D) ou numa encosta (3D). O programa Hydrus ${ }^{\circledR}$ é um modelo de elementos finitos, que numericamente resolve a equação de Richards para o fluxo saturado/insaturado da água no solo e a equação de convecção-dispersão para o transporte de solutos.

O objetivo deste trabalho é apresentar uma modelagem alternativa para estimar as variações de umidade num perfil de solo heterogêneo e bem drenado em função do tempo. Como metodologia, utiliza-se uma abordagem compartimental em que para a redistribuição da água no solo é feita uma analogia com os modelos de competição em dinâmica de populações (Murray 2002; Edelstein-Keshet 2005). A motivação para esta abordagem vem de observar o movimento da água entre os horizontes de um perfil de solo como o efeito de forças em tensão querendo sempre mais potência (água). Assim como duas espécies de uma comunidade disputam os mesmos recursos do ambiente numa competição interespecífica, como, por exemplo, as raízes de plantas competindo por água e nutrientes, os diferentes horizontes de um perfil de solo se relacionam de forma contínua em busca de um aumento no teor de umidade. Considerando esta analogia, entre as forças que resultam na dinâmica da água no solo, destacam-se o potencial gravitacional e o potencial matricial. A acurácia da metodologia proposta é avaliada a partir dos resultados obtidos usando o programa gratuito Hydrus-1D (Simunek et al. 2009). Geralmente, os modelos de competição em dinâmica populacional consistem de um sistema de equações diferenciais ordinárias de primeira ordem, em que as variáveis dependentes são os níveis populacionais das espécies e a variável independente é o tempo. O clássico método de Runge-Kutta de $4^{a}$ ordem é um dos mais usados para a resolução 
numérica destes sistemas de equação diferenciais, por ser uma combinação de simplicidade, alta precisão e economia (Burden \& Faires 2011).

\section{Metodologia}

\section{Formulação matemática clássica}

O movimento da água no solo ocorre a partir da diferença de potencial total da água $\psi \mathrm{e}$ se dá no sentido do maior potencial para o menor. A equação de Darcy-Buckingham relaciona o fluxo da água $(q)$ com a variação de potencial total num meio poroso isotrópico e não saturado, que para o caso de um fluxo unidimensional vertical pode ser expressa pela seguinte equação

$$
q_{z}=-k(\theta) \frac{\partial \psi}{\partial z}
$$

onde $z$ denota a dimensão vertical e $k(\theta)$ condutividade hidráulica, função da umidade $\theta$. Para solo não saturado e desconsiderando o potencial osmótico, o potencial total é a soma do potencial matricial $\left(\psi_{m}\right)$ com o potencial gravitacional $\left(\psi_{g}\right)$.

O potencial matricial representa a força de adsorção da água nas partículas sólidas, sendo o componente de potencial total que mais tem sido avaliado devido a sua importância para o manejo da irrigação e nos estudos do movimento da água no solo. O potencial gravitacional depende principalmente da posição de uma unidade volumétrica de água dentro do campo gravitacional, em relação a um referencial, aqui sendo obtido medindo-se a distância vertical do ponto de interesse no solo até a superfície do solo. Considerando os componentes de potencial matricial e gravitacional, a Equação (1) pode ser escrita da seguinte forma

$$
q_{z}=-\left(D(\theta) \frac{\partial \theta}{\partial z}+k(\theta)\right)
$$

Onde $D(\theta)=k(\theta) \frac{\partial \psi_{m}}{\partial \theta}$ é a difusidade.

A umidade do solo varia ao longo do tempo respeitando a equação da continuidade

$$
\frac{\partial \theta}{\partial t}=-\frac{\partial q_{z}}{\partial z}
$$

onde não estão sendo considerados os fluxos de entrada (fonte de precipitação ou de irrigação) e de saída (fonte de evapotranspiração). Combinando as Equações (2) e (3), chega-se na equação de Richards baseada na variação de umidade no solo.

$$
\frac{\partial \theta}{\partial t}=\frac{\partial}{\partial z}\left(D(\theta) \frac{\partial \theta}{\partial z}+k(\theta)\right) .
$$

Para ser resolvida, a Equação (4) deve estar sujeita á condição inicial e condições de contorno. Por ser uma equação diferencial parcial não-linear, sua solução numérica não é facilmente obtida. A seguir, apresenta-se uma alternativa para a modelagem do escoamento transiente da água em solo não-saturado unidimensional que, por se tratar de um sistema de equações diferenciais ordinárias de primeira ordem, possui uma solução numérica mais estável que a Equação (4). 


\section{Modelagem matemática proposta}

O clássico modelo de competição entre espécies consiste de um sistema de equações diferenciais ordinárias, em que as variáveis dependentes são os níveis populacionais das espécies e a variável independente é o tempo, conforme pode ser visto em Edelstein-Keshet (2005).

$$
\left\{\begin{array}{l}
\frac{d x}{d t}=a x\left(1-\frac{x}{k_{1}}\right)-b x y \\
\frac{d y}{d t}=c y\left(1-\frac{y}{k_{2}}\right)-d x y .
\end{array}\right.
$$

O termo $x y$ na Equação (5) representa a probabilidade de um encontro ocorrer entre as populações $x$ e $y$, dado que ambas as espécies se movem aleatoriamente e estão uniformemente distribuídas em seu habitat. A forma dessa taxa de encontro é derivada da lei de ação das massas que, em seu contexto original, afirma que a taxa de colisões moleculares de duas espécies químicas em um gás diluído ou solução é proporcional ao produto das duas concentrações.

No processo de redistribuição da água no solo existe uma "competição" entre os níveis de água presentes nas diferentes camadas do solo. $\mathrm{O}$ modelo físico proposto é do tipo compartimental e a motivação para esta opção vem do fato que as camadas de solo sofrem diferentes influências. $O$ contato entre as camadas faz com que ocorra uma perda de água para a camada com maior demanda, sentido do maior potencial para o menor, ocorrendo uma flutuação nos valores da umidade. Neste trabalho, propõe-se que a taxa de variação da umidade na i-ésima camada contabilize esta perda através de parcelas do tipo

$$
-k\left(\theta_{i}\right) \theta_{i}(t)\left(1-\frac{\theta_{i+1}(t)}{\theta_{s_{i+1}}}\right),
$$

sendo $\theta_{i+1}$ a umidade da camada inferior adjacente e $\theta_{s_{i+1}}$ sua umidade de saturação. A Equação (6) vai realizar o movimento da água entre as camadas de um solo bem drenado, quantificando a perda de água da camada $i$ em decorrência do ganho de umidade da camada inferior adjacente $i+1$. Neste solo bem drenado, a redistribuição da água no solo é caracterizada pelo processo de drenagem interna onde a água da camada superficial, mais úmida, tende a ser distribuída para as camadas inferiores, mais secas, do perfil. Aqui, não se considera a ascensão capilar, ou seja, o fenômeno pela qual ao invés da água drenar pela força da gravidade, ela sobe, o que pode acontecer nas proximidades dos lençóis freáticos. Na Equação (6), observa-se, ainda, que quanto maior a umidade na profundidade $i+1$ menor é a perda de umidade da $i$-ésima camada.

Para se modelar a taxa de variação de $\theta_{i}(t)$ são analisadas as formas de perdas e ganhos de umidade na profundidade $i$. Destaca-se o primeiro compartimento, em que há contato com a atmosfera. Nele ocorre ganho de água por precipitação (chuva e/ou irrigação) representado por $f$, cuja unidade é do tipo $\left(\frac{\text { volume }}{(\text { área)(tempo) }}\right)$. Também ocorre perda por evapotranspiração representada por $\epsilon$, com unidade de medida do mesmo tipo que $f$; além de ocorrer perda de água pelo processo de redistribuição. Por sua vez, a segunda camada, que tem contato com a primeira, ganha a água que a primeira perdeu por redistribuição e perde água para a terceira, também pelo processo de redistribuição. Esse processo se dá enquanto houver camadas de solo no perfil considerado. Note que a escolha da abordagem compartimental para a modelagem permite analisar o perfil do solo em cada profundidade. Considera-se que na i-ésima camada a umidade está uniformemente distribuída, ou seja, $\theta_{i}(t)$ é a mesma em qualquer volume de solo dentro de uma mesma camada de espessura $h_{i}$.

Seguindo o processo descrito acima, propõem-se o seguinte sistema de equações diferencias ordinárias de primeira ordem para modelar a variação de umidade num perfil de solo, considerando três camadas. 


$$
\left\{\begin{array}{c}
\frac{d \theta_{1}}{d t}=\frac{f}{\theta_{s_{1}} h_{1}}-\frac{\epsilon}{\theta_{s_{1}} h_{1}}-\frac{k_{1}\left(\theta_{1}\right)}{\theta_{s_{1}} h_{1}} \theta_{1}\left(1-\frac{\theta_{2}}{\theta_{s_{2}}}\right) \\
\frac{d \theta_{2}}{d t}=\frac{k_{1}\left(\theta_{1}\right)}{\theta_{s_{1}} h_{1}} \theta_{1}\left(1-\frac{\theta_{2}}{\theta_{s_{2}}}\right)-\frac{k_{2}\left(\theta_{2}\right)}{\theta_{s_{2}} h_{2}} \theta_{2}\left(1-\frac{\theta_{3}}{\theta_{s_{3}}}\right) \\
\frac{d \theta_{3}}{d t}=\frac{k_{2}\left(\theta_{2}\right)}{\theta_{s_{2}} h_{2}} \theta_{2}\left(1-\frac{\theta_{3}}{\theta_{s_{3}}}\right)-\frac{k_{3}\left(\theta_{3}\right)}{\theta_{s_{3}} h_{3}} \theta_{3}\left(1-\frac{\theta_{3}}{\theta_{s_{3}}}\right)
\end{array}\right.
$$

No sistema de equações (7), as razões $\frac{f}{\theta_{s_{1}} h_{1}}, \frac{\epsilon}{\theta_{s_{1}} h_{1}} \mathrm{e} \frac{k_{i}\left(\theta_{i}\right)}{\theta_{s_{i}} h_{i}}$ representam, respectivamente, o volume de água que entra num volume total de poros por unidade de tempo, um volume de água que sai de um volume total de poros por unidade de tempo e a passagem de um volume de água por um volume total de poros por unidade de tempo. Neste trabalho, o sistema (7) sujeito a uma condição inicial é resolvido através do método Runge-Kutta de $4^{\mathrm{a}}$ ordem (Burden \& Faires 2011), utilizando um tamanho de passo fixo $\Delta t=0.01$. Além disso, para o teste numérico proposto, as fontes $f$ e $\epsilon$ são nulas.

\section{Teste numérico}

A performance da modelagem proposta é testada simulando o fluxo de água em um solo seco, a partir de um experimento de infiltração em larga escala conduzido por Zadeh et al. (2007). Os resultados obtidos pelo modelo são comparados com os resultados obtidos a partir do programa gratuito Hydrus-1D.

O simulador Hydrus-1D resolve a equação de Richards de forma mista através do método dos elementos finitos com um esquema implícito proposto em Celia et al. (1990). Esta forma mista da equação de Richards é caracterizada por envolver as variáveis conteúdo volumétrico da água $(\theta)$ e o potencial matricial $\left(\psi_{m}\right)$.

As propriedades hidráulicas do solo são necessárias para descrever o conteúdo da água $\theta$ e a condutividade hidráulica $k(\theta)$ em solo não saturado. Aqui, utiliza-se o modelo de Mualemvan Genuchten (van Genuchten 1980), dado pelas seguintes expressões.

$$
\begin{gathered}
S_{e}(\theta)=\frac{\theta-\theta_{r}}{\theta_{s}-\theta_{r}} \begin{cases}\frac{1}{\left(1+\left|\alpha \psi_{m}\right|^{n}\right)^{m}}, & \psi_{m}<0 \\
1, & \psi_{m} \geq 0\end{cases} \\
k(\theta)= \begin{cases}k_{s} S_{e}^{0.5}\left[1-\left(1-s_{e}^{\frac{1}{m}}\right)^{m}\right]^{2}, & \psi_{m}<0 \\
k_{s}, & \psi_{m} \geq 0\end{cases}
\end{gathered}
$$

onde $S_{e}$ é o grau de saturação, $k_{s}$ é a condutividade hidráulica em solo saturado, $\theta_{r}$ e $\theta_{s}$ são as umidades residual e de saturação, respectivamente, e $n$ e $m$ são parâmetros adimensionais relacionados com o tamanho e a distribuição dos poros, com $m=1-\frac{1}{n}$ e $n>1$.

\section{Resultados e Discussão}

O modelo é testado considerando um experimento que simula o processo de infiltração da água em um solo seco, onde são estimados os valores de umidade em quatro profundidades diferentes: 5, 10, 15 e $20 \mathrm{~cm}$. Os parâmetros do modelo de Mualem-van Genuchten usados na simulação foram $\theta_{r}=0.062, \theta_{s}=0.46, \alpha=0.037 \mathrm{~cm}^{-1}, n=1.67$ e $k_{s}=0.44 \mathrm{~cm} \cdot \mathrm{h}^{-1}$ (Zadeh 2011). Caviedes-Voullieme et al. (2013) sugeriram o uso de uma condutividade de saturação $k_{s}=0.34 \mathrm{~cm} . h^{-1}$, para uma melhor correlação com os dados mensurados. Considera-se uma condição inicial uniforme de $\theta_{i}=0.063\left(S_{e}=0.0025\right)$, simulando um solo seco. 
Para a aplicação do programa Hydrus-1D, foi prescrito $\psi_{m}=0 \mathrm{~cm}$ para a condição de contorno na superfície do solo e uma drenagem livre como condição de contorno inferior. Além disso, o domínio teve comprimento de $60 \mathrm{~cm}$ discretizado com 150 elementos uniformes de tamanho $\Delta z=0.4 \mathrm{~cm}$, um tamanho inicial de passo de tempo de $\Delta t_{0}=0.01 \mathrm{~s}$ e um tamanho de passo máximo de $\Delta \mathrm{t}_{\max }=10 \mathrm{~s}$.

As Figuras 1-2 apresentam as curvas de umidade nas quatro profundidades analisadas para um período de 24 horas, obtidas pela modelagem proposta e considerando, respectivamente, $k_{s}=0.34 \mathrm{~cm} \cdot \mathrm{h}^{-1}$ e $k_{s}=0.44 \mathrm{~cm} \cdot \mathrm{h}^{-1}$. As figuras mostram o aumento do teor de umidade em cada camada, com uma capacidade de suporte dada pela umidade de saturação $\left(\theta_{s}=0.46\right)$ e ocorrendo de forma mais rápida para $k_{s}=0.44 \mathrm{~cm} \cdot \mathrm{h}^{-1}$, como esperado.

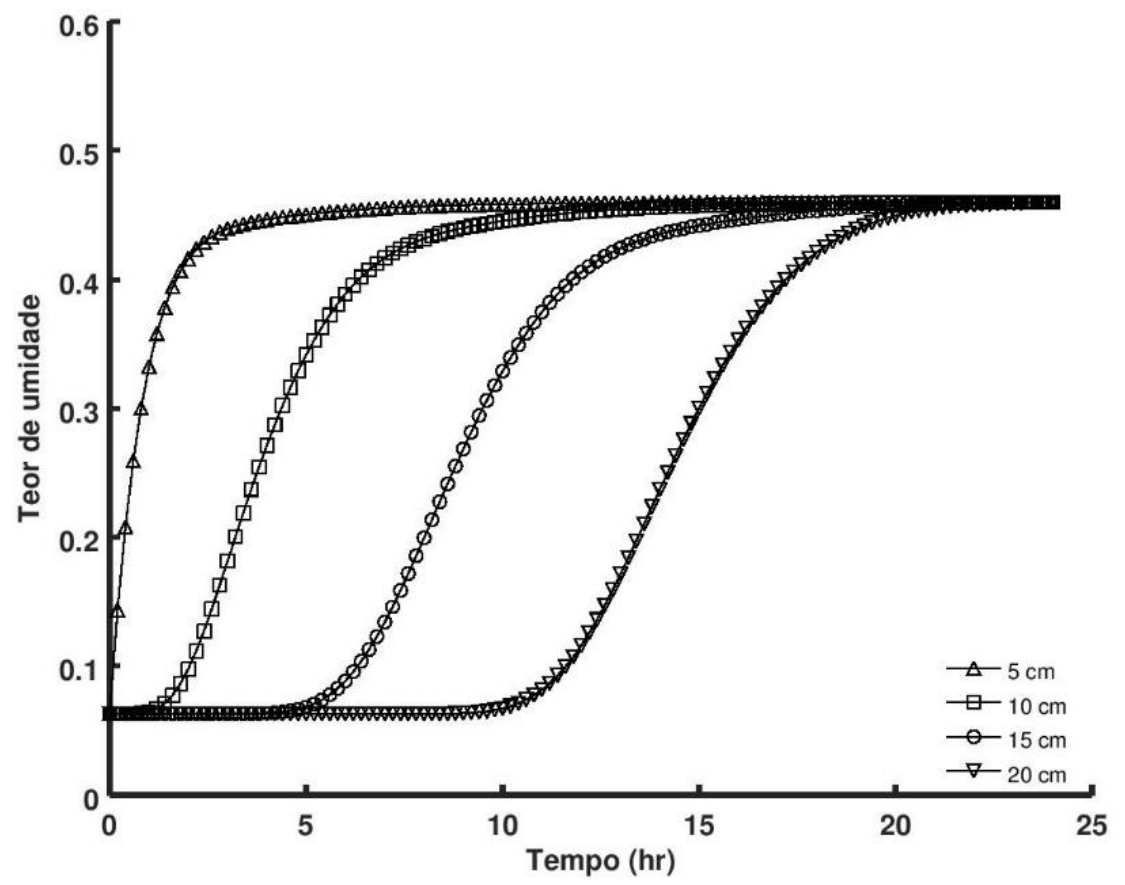

Figura 1. Simulação numérica do conteúdo da água no solo com $k_{s}=0.34 \mathrm{~cm} \cdot \mathrm{h}^{-1}$.

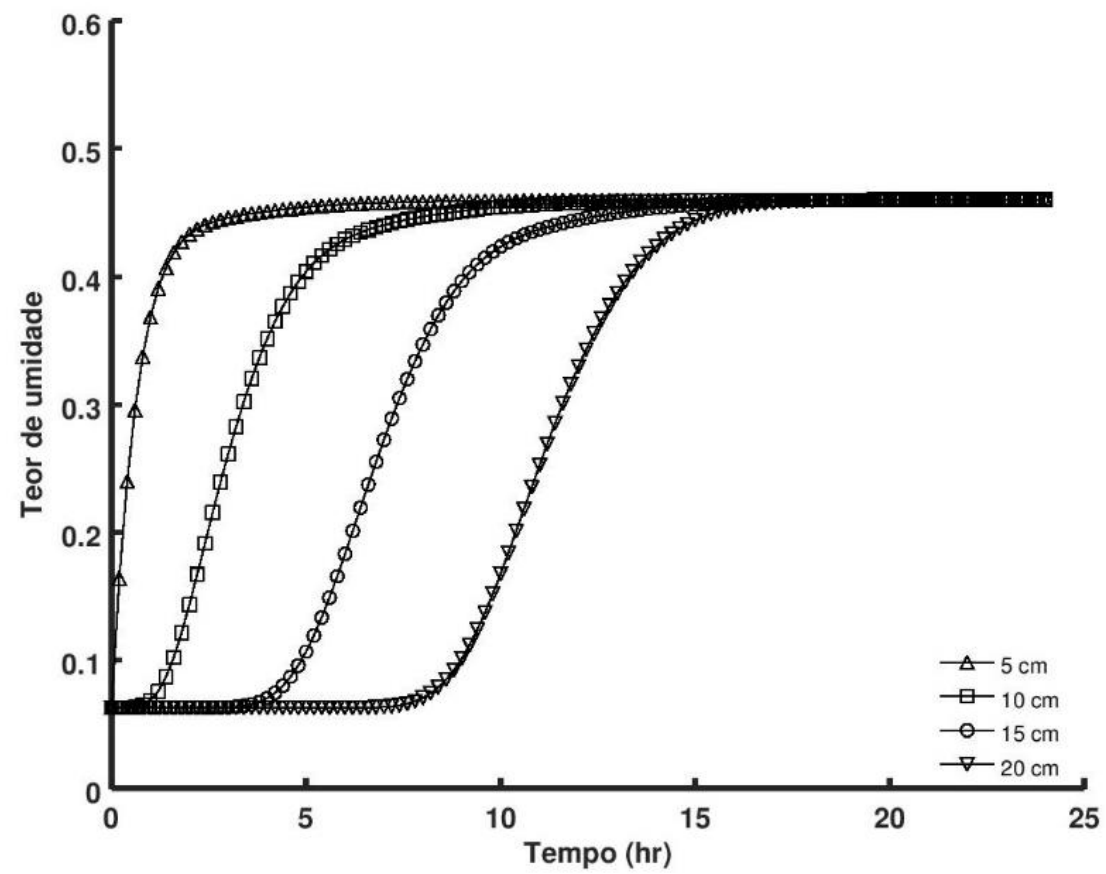

Figura 2. Simulação numérica do conteúdo da água no solo com $k_{s}=0.44 \mathrm{~cm} \cdot \mathrm{h}^{-1}$. 
As Figuras 3-6 ilustram o comportamento da umidade para $k_{s}=0.34 \mathrm{~cm} . \mathrm{h}^{-1}$, avaliando separadamente cada profundidade e comparando a modelagem proposta com os resultados obtidos pelo programa Hydrus-1D. O modelo proposto apresentou resultados similares aos obtidos pelo programa Hydrus-1D, sendo que no Hydrus-1D houve um atraso no início da variação de umidade no solo em relação à modelagem proposta. Observa-se, ainda, que as curvas de umidade obtidas através do sistema (7) possuem um comportamento mais suave.

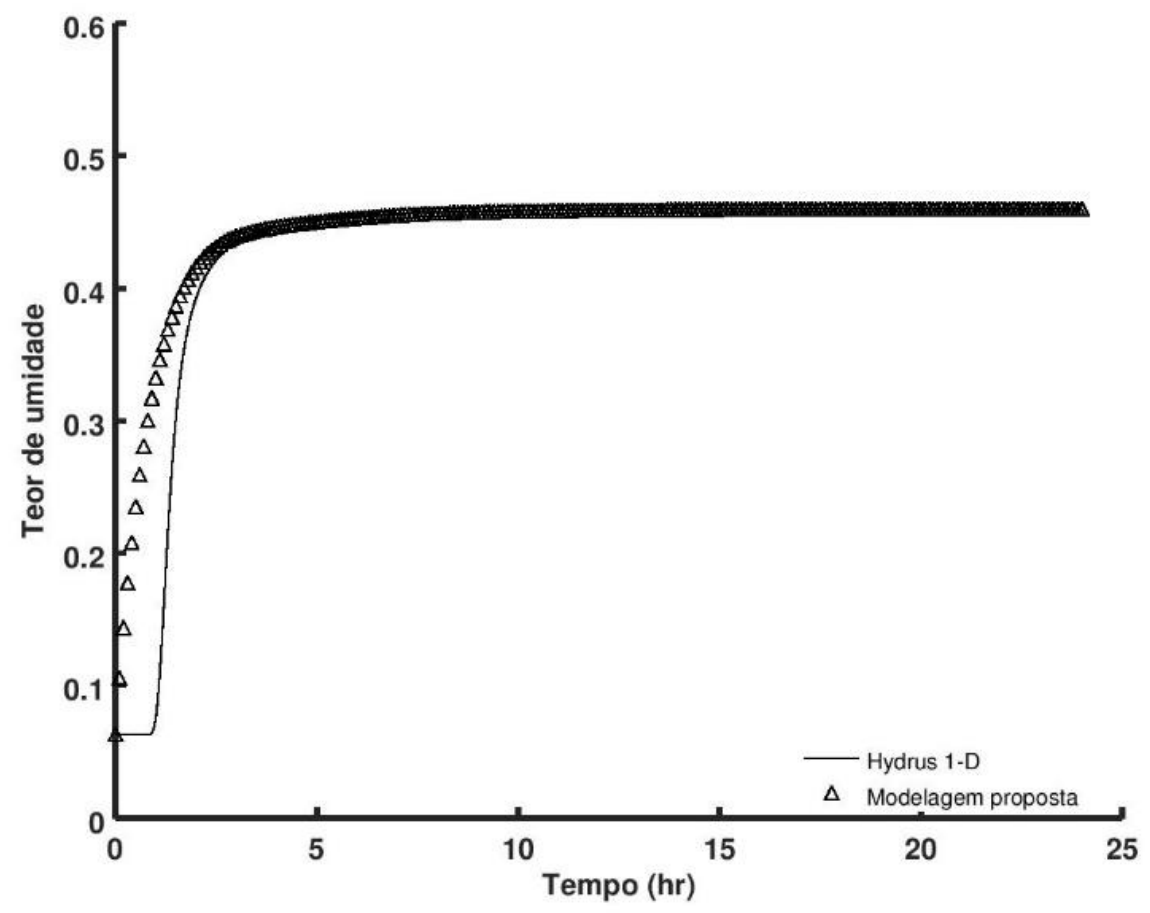

Figura 3. Simulação numérica do conteúdo da água na profundidade de $5 \mathrm{~cm}$ via modelagem proposta e Hydrus-1D.

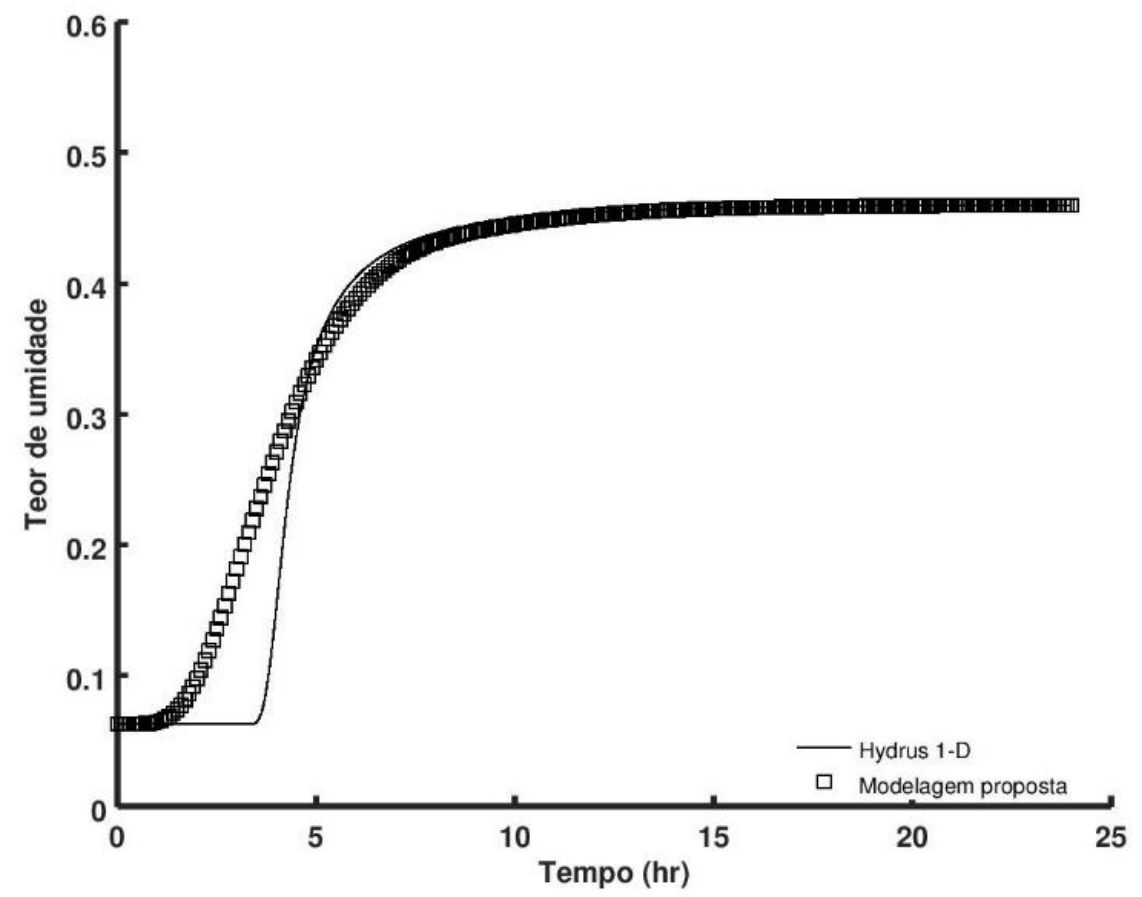

Figura 4. Simulação numérica do conteúdo da água na profundidade de $10 \mathrm{~cm}$ via modelagem proposta e Hydrus-1D. 


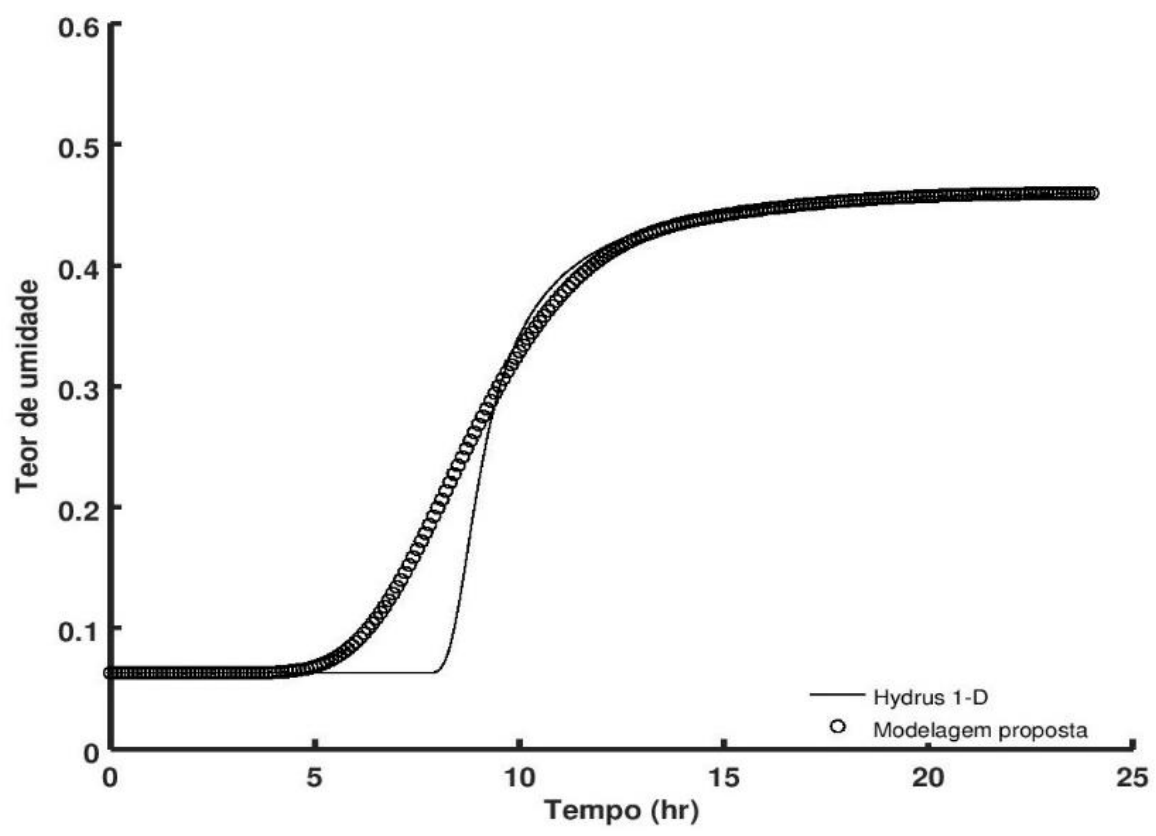

Figura 5. Simulação numérica do conteúdo da água na profundidade de $15 \mathrm{~cm}$ via modelagem proposta e Hydrus-1D.

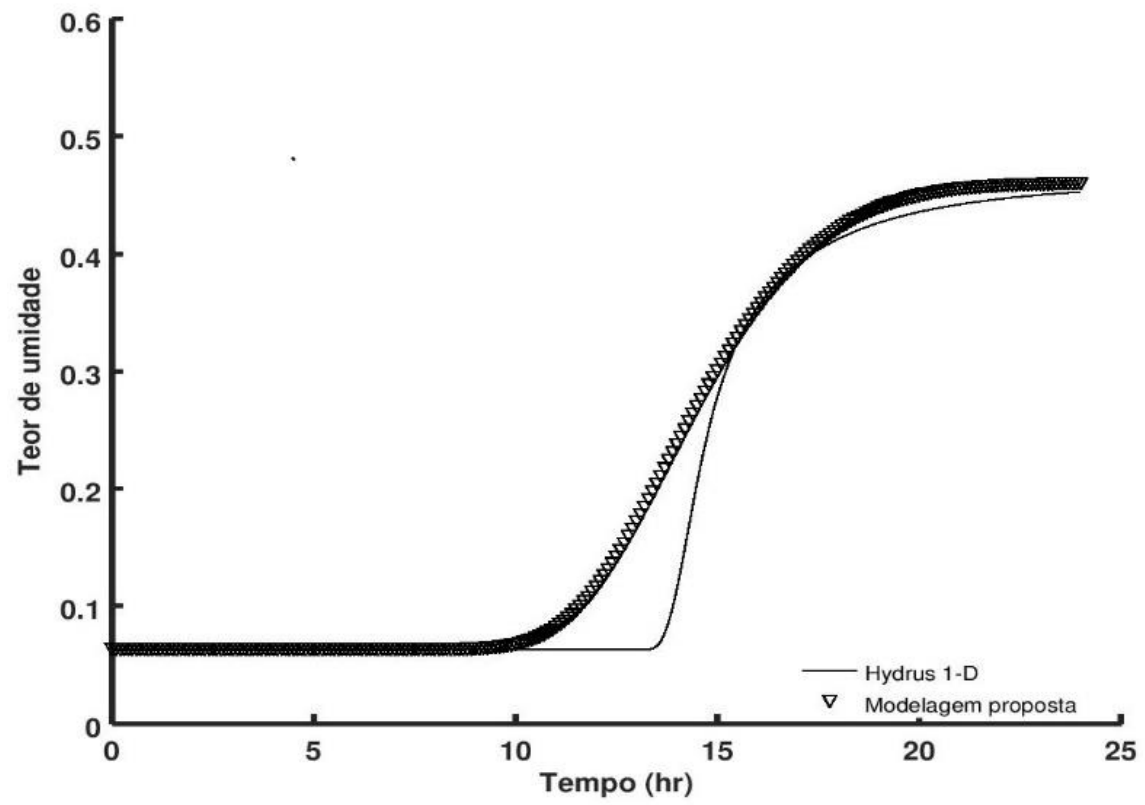

Figura 6. Simulação numérica do conteúdo da água na profundidade de $20 \mathrm{~cm}$ via modelagem proposta e Hydrus-1D.

\section{Considerações finais}

O presente trabalho teve como proposta apresentar e testar uma modelagem alternativa para a dinâmica da água em um solo heterogêneo, bem drenado e para um determinado intervalo de tempo. A metodologia proposta consistiu em um modelo compartimental, formado por um sistema de equações diferenciais ordinárias de primeira ordem que se caracteriza por possuir uma solução numérica mais estável do que a altamente não-linear equação diferencial parcial de Richards. Sob o ponto de vista econômico, a metodologia implementada baseada no 
clássico método de Runge-Kutta de $4^{\mathrm{a}}$ ordem representa um ganho significativo quando comparada com as tecnologias que podem ser usadas para mensurar a umidade do solo, tais como TDR (Time Domain Reflectometry) e lisímetro.

O modelo proposto foi avaliado a partir de um teste numérico, cuja performance foi comparada com o já conceituado e bastante referenciado programa de uso gratuito Hydrus-1D. Através das figuras apresentadas no presente texto, observa-se que a modelagem proposta conseguiu representar de forma qualitativa o fenômeno do escoamento transiente da água em um solo seco unidimensional. Em comparação com o Hydrus-1D, observa-se que as curvas de umidade obtidas pelo modelo proposto se comportam de forma mais suave. Isso pode ser explicado pela natureza distinta entre a modelagem matemática apresentada e a tradicional formulação matemática dada pela equação de Richards. Nota-se, ainda, que o teor de umidade em cada profundidade começa a aumentar em instantes diferentes para cada método utilizado. A simulação realizada com o modelo compartimental proposto iniciou de forma suavemente antecipada a variação de umidade em cada profundidade, quando comparada com a simulação numérica realizada com o Hydrus-1D. O comportamento assintótico é similar em ambos os métodos.

Vale salientar que, para uma completa validação, o modelo proposto deve ser avaliado para diferentes tipos de solos, em relação à textura, estrutura e conteúdo de matéria orgânica, considerando experimentos laboratoriais e em campo controlado. Tais experimentos estão em fase de elaboração na Fazendinha Agroecológica, localizada no município de Seropédica-RJ, e nos laboratórios de Física do Solo do Departamento de Solos da UFRuralRJ e serão assuntos de futuras publicações.

\section{Agradecimentos}

Ao Conselho Nacional de Desenvolvimento Científico e Tecnológico (CNPq) e à Universidade Federal Rural do Rio de Janeiro (UFRRJ). Aos avaliadores anônimos pela revisão crítica do manuscrito.

\section{Referências}

Bornhöft D. (1994) A simulation model for the description of the one-dimensional vertical soil water flow in the unsaturated zone. Ecological Modelling, 75/76: 269-278. Doi: 10.1016/03043800(94)90024-8

Burden R.L. \& Faires J.D. (2011) Numerical Analysis. Brooks/Cole: MA. 872 p.

Caviedes-Voullieme D., Garcia-Navarro P. \& Murillo J. (2013) Verification, conservation, stability and efficiency of a finite volume method for the 1D Richards equation. Journal of Hydrology, 480: 69-84. Doi: 10.1016/j.jhydrol.2012.12.008

Celia M.A., Bouloutas E.T. \& Zarba R.L. (1990) A general mass-conservative numerical solution for the unsaturated flow equation. Water Resources Research, 26(7): 1483-1496. Doi: 10.1029/WR026i007p01483

Dardanelli J.L., Ritchie J.T., Calmon M., Andriani J.M. \& Collino D.J. (2004) An empirical model for root water uptake. Field Crops Research, 87: 59-71. Doi: 10.1016/j.fcr.2003.09.008

Edelstein-Keshet L. (2005) Mathematical Models in Biology. SIAM Classics in Applied Mathematics. 586 p.

Eymard R., Gutnic M. \& Hilhorst D. (1999) The finite volume method for Richards equation. Computational Geosciences, 3(3-4): 259-294. Doi: 10.1023/A:1011547513583

Forsyth P.A., Wu Y.S. \& Pruess K. (1995) Robust numerical methods for saturated-unsaturated flow with dry initial conditions in heterogeneous media. Advances in Water Resources, 18(1): 25-38. Doi: 10.1016/0309-1708(95)00020-J

Lai W. \& Ogden F.L. (2015) A mass-conservative finite volume predictor-corrector solution of the 1D Richards' equation. Journal of Hydrology, 523: 119-127. Doi: 10.1016/j.jhydrol.2015.01. 053 
Murray J.D. (2002) Mathematical Biology - An Introduction. $3^{\circ}$ edition. Springer-Verlag: New York. $553 \mathrm{p}$.

Reichardt K. \& Timm L.C. (2004) Solo, planta atmosfera: conceitos, processos e aplicações. Barueri, SP: Manole. 478 p.

Richards L.A. (1931) Capillary conduction of liquids through porous mediums. Physics, 1(5): 318-333. Doi: 10.1063/1.1745010

Simunek J., Sejna M., Saito H., Sakai M. \& van Genuchten M.T. (2009) The HYDRUS-1D Software Package for Simulating the One-dimensional Movement of Water, Heat, and Multiple Solutes in Variably-saturated Media. Department of Environmental Sciences, University of California Riverside, Riverside, CA.

Simunek J., Sejna M. \& Van Genuchten M.T. (1999) HYDRUS-2D: simulating water flow and solute transport in two-dimensional variably saturated media, Technical Report, IGWMC, Golden, CO, USA.

Takeuchi J., Kawachi T., Imagawa C., Buma N., Unami K. \& Maeda S. (2010) A physically based fvm watershed model fully coupling surface and subsurface water flows. Paddy Water Environment, 8: 145-156. Doi: 10.1007/s10333-009-0193-7

Vale I.G., Delgado A.R.S. \& Ventura S.D. (2016) Sistemas de viabilidade agrícola sustentáveis. Irriga, 21(2): 342-351. Doi: 10.15809/irriga.2016v21n2p342-351

Van Genuchten M.Th (1980) A closed-form equation for predicting the hydraulic conductivity of unsaturated soils. Soil Science Society of America Journal, 44: 892-898. doi: 10.2136/sssaj1980. $03615995004400050002 x$

Zadeh K.S. (2011) A mass-conservative switching algorithm for modeling fluid flow in variably saturated porous media. Journal of Computational Physics, 230(3): 664-679. Doi: 10.1016/j.jc p.2010.10.011

Zadeh K.S., Shirmohammadi A., Montas H.J. \& Felton G. (2007) Evaluation of infiltration models in contaminated landscape. Journal of Environmental Science and Health Part A, 42(7): 983988. Doi: 10.1080/10934520701373000

Zambra C.E., Dumbser M., Toro E.F. \& Moraga N.O. (2012) A novel numerical method of highorder accuracy for flow in unsaturated porous media. International Journal for Numerical Methods Engineering, 89: 227-240. Doi: 10.1002/nme.3241 